\title{
ON THE STABILITY OF SOBOLEV SPACES WITH ZERO BOUNDARY VALUES
}

\author{
LARS INGE HEDBERG and TERO KILPELÄINEN
}

\begin{abstract}
In this note we characterize the open sets $\Omega$ for which

$$
W^{1, p}(\Omega) \cap \bigcap_{q<p} W_{0}^{1, q}(\Omega)=W_{0}^{1, p}(\Omega) .
$$

We apply our result to establish the stability of the first eigenvalues of the $p$-Laplacians with varying $p$.
\end{abstract}

\section{Introduction}

Let $\Omega$ be a bounded open set in $\mathrm{R}^{n}$. By $W^{1, q}(\Omega)$ we denote the usual Sobolev space of those $p$-integrable functions $u$ whose first distributional derivatives are also $p$-integrable in $\Omega$. The closure in $W^{1, p}(\Omega)$ of compactly supported smooth functions on $\Omega$ is denoted by $W_{0}^{1, p}(\Omega)$. The space $W_{0}^{1, p}(\Omega)$ is often vaguely described as the collection of those functions from $W^{1, p}(\Omega)$ that "vanish on the boundary $\partial \Omega$ ". This statement has been made precise in the Havin-Bagby Theorem (see Theorem 2.1 below).

The purpose of this note is to find properties of $\Omega$ that would allow us to conclude that

$$
W^{1, p}(\Omega) \cap \bigcap_{q<p} W_{0}^{1, q}(\Omega)=W_{0}^{1, p}(\Omega) .
$$

This question has arisen in stability problems for certain partial differential equations; see e.g. [11] or Section 5 below. Of course the inclusion " $\supset$ " in (1.1) always holds as is seen by Hölder's inequality. Moreover, we infer from the Havin-Bagby theorem 2.1 that (1.1) holds for $p>n$. If $1<p \leq n$, there are domains $\Omega$ for which (1.1) fails to hold: for instance, let $\Omega=B \backslash C$, where $B$ is a ball and $C$ a compact subset of $B$ such that the $p$-capacity (see

\footnotetext{
* The first-named author gratefully acknowledges support from the Centre de Mathématiques et de Leurs Applications, École Normale Supérieure de Cachan. The research of the second author is financed by the Academy of Finland.

Received March 11, 1997; in the revised form September 22, 1997.
} 
Section 2) of $C$ is positive but the Hausdorff dimension of $C$ is $n-p$ (It is well known that such sets exist; see [1, Ch. 5] or 11, 7.1] for explicit constructions.)

As the above example indicates, a thickness condition on the complement of $\Omega$ must be imposed in order to obtain (1.1). In this paper we give two approaches to treat the problem. The first one is rather elementary and self contained. It gives a sufficient condition for $\Omega$ to enjoy the property (1.1). This condition can be spelled out in terms of Hausdorff measure densities which makes it rather usable in practice.

The second approach uses more advanced results of potential theory. The resulting theorem gives a complete solution to problem (1.1), but the condition is somewhat difficult to verify. Hence we decided to present both methods here.

For the first approach we introduce a class of open sets whose complement is quasi everywhere sufficiently dense: We use the capacity density; the upper p-capacity density of a set $E$ at the point $x$ is the number

$$
\overline{\operatorname{dens}}_{p}(E, x)=\limsup _{r \rightarrow 0} \frac{\operatorname{cap}_{p}(E \cap B(x, r), B(x, 2 r))}{r^{n-p}}
$$

and the class of open sets satisfying the p-density condition is

$\Omega_{p}^{\text {dens }}=\left\{\Omega \subset \mathrm{R}^{n}\right.$ : for $p$-q.e. $x \in \partial \Omega$ there is $q<p$ such that $\left.\overline{\operatorname{dens}}_{q}(\complement \Omega, x)>0\right\}$;

for the precise definitions the reader is referred to Section 2.

Observe that $q<p$ may depend on $x$ in the definition for $\Omega_{p}^{\text {dens }}$. In Lemma 3.1 below we describe the class $\Omega_{p}^{\text {dens }}$ in terms of Hausdorff measure densities.

There is an abundance of domains that satisfy the density condition. For instance, one easily infers that smooth or Lipschitz domains and domains satisfying an exterior cone condition are in $\Omega_{p}^{\mathrm{dens}}$ for each $p$. Moreover, if $p>n$, then $\Omega_{p}^{\text {dens }}$ contains all open sets. If $\Omega \in \Omega_{p}^{\text {dens }}$ and $K$ is a compact set of $p$-capacity zero, then $\Omega \backslash K \in \Omega_{p}^{\text {dens }}$.

Our first result states that if a bounded open set $\Omega$ satisfies the $p$-density condition, then (1.1) holds:

1.2. Theorem. Let $u \in W^{1, p}(\Omega)$ and $\Omega \in \Omega_{p}^{\mathrm{dens}}$. If $u \in W_{0}^{1, q}(\Omega)$ for each $q<p$, then $u \in W_{0}^{1, p}(\Omega)$.

We need more notation for the second approach. Let

$$
E_{p}=\{x \in \complement \Omega: \complement \Omega \text { is } q \text {-thin for all } q<p\}
$$

and 


$$
B_{p}=\{x \in \complement \Omega: \complement \Omega \text { is } q \text {-thick for some } q<p\}
$$

(see definitions in Section 2 below). Then

$$
\complement \Omega \backslash E_{p}=B_{p}
$$

and the set of $p$-admissible open sets is

$$
\begin{aligned}
\Omega_{p} & =\left\{\Omega \subset \mathrm{R}^{n}: \complement \Omega \backslash E_{p} \text { is } p \text {-thick } p \text {-q.e. on } \partial \Omega\right\} \\
& =\left\{\Omega \subset \mathrm{R}^{n}: B_{p} \text { is } p \text {-thick } p \text {-q.e. on } \partial \Omega\right\} .
\end{aligned}
$$

1.3. Theorem. Let $\Omega$ be a bounded open set in $\mathrm{R}^{n}$. Then

$$
W^{1, p}(\Omega) \cap \bigcap_{q<p} W_{0}^{1, q}(\Omega)=W_{0}^{1, p}(\Omega)
$$

if and only if $\Omega \in \Omega_{p}$.

By the Kellogg property we have:

1.4. Corollary. Let $\Omega$ be a bounded open set in $\mathrm{R}^{n}$. If $E_{p} \cap \partial \Omega$ is of p-capacity zero, then

$$
W^{1, p}(\Omega) \cap \bigcap_{q<p} W_{0}^{1, q}(\Omega)=W_{0}^{1, p}(\Omega) .
$$

Note that $E_{p} \cap \partial \Omega$ is of $q$-capacity zero for each $q<p$ by the Kellogg property. Moreover, $E_{p} \cap \partial \Omega$ is of $p$-capacity zero for instance when $\Omega$ is regular for the $q$-Laplacian Dirichlet problem for some $q<p$, because then $\lceil\Omega$ is $q$-thin nowhere on $\partial \Omega[6]$.

The problem (1.1) seems to be rather untouched in the literature. Naturally, (1.1) is known to be true for Lipschitz domains. Martio informed us that he and $\mathrm{Li}$ [9] have proven the equality (1.1) for domains $\Omega$ whose complement is uniformly $p$-fat (see 3.6 below; see also [7]). The key point in their argument is that then, according to Lewis' nice paper [8], a uniform Hardy inequality holds for $C_{0}^{\infty}(\Omega)$ functions.

Problem (1.1) arose in the work [11], where Lindqvist studied the convergence of the first eigenvalues and eigenfunctions of the $p$-Laplacian operators with varying $p$. We apply our analysis of Sobolev spaces to these convergence problems; see section 5 for a more thorough discussion.

Acknowledgement. We thank Peter Lindqvist for proposing this problem and for stimulating discussions. 


\section{Preliminaries}

In this section we give the necessary definitions and state the auxiliary results for the reader's convenience.

We use the $p$-capacity that is naturally connected with the Sobolev space $W^{1, p}$. The Sobolev p-capacity of the set $E \subset \mathrm{R}^{n}$ is the number

$$
C_{p}(E)=\inf _{u} \int_{\mathrm{R}^{n}}\left(|u|^{p}+|\nabla u|^{p}\right) d x,
$$

where the infimum is taken over all $u \in W^{1, p}\left(\mathrm{R}^{n}\right)$ such that $u \geq 1$ on an open neighborhood of $E$.

As usual, we say that a property holds $p$-quasi everywhere (abbreviated $p$ q.e.) on a set $A$ if it holds at each $x \in A \backslash E$, where $C_{p}(E)=0$. Recall that for $p>n$ each nonempty set has positive $p$-capacity. For relations to Hausdorff measures see 2.2 below.

A set $E \subset \mathrm{R}^{n}$ is called $p$-thin at $x$ if

$$
\int_{0}^{1}\left(\frac{C_{p}(E \cap B(x, r))}{r^{n-p}}\right)^{1 /(p-1)} \frac{d r}{r}<\infty .
$$

If $E$ is not $p$-thin at $x$ it is $p$-thick at $x$. Write

$$
e_{p}(E)=\left\{x \in \mathbf{R}^{n}: E \text { is } p \text {-thin at } x\right\}
$$

and

$$
b_{p}(E)=\left\{x \in \mathrm{R}^{n}: E \text { is } p \text {-thick at } x\right\} .
$$

Then the Kellogg property holds, i.e.

$$
C_{p}\left(e_{p}(E) \cap E\right)=0
$$

[1, Cor. 6.3.17]. Further, one easily shows that $e_{p}(E) \subset e_{q}(E)$ if $1 \leq q \leq p$; see $[1,6.5 .8]$.

Each function $u \in W^{1, p}(\Omega)$ has a p-quasi continuous representative $v$ (i.e. for each $\varepsilon>0$ there is an open set $G$ with $C_{p}(G)<\varepsilon$ such that the restriction of $v$ to $\Omega \backslash G$ is continuous). The quasi continuous representative is unique in the sense that two $p$-quasi continuous functions that agree almost everywhere coincide $p$-quasi everywhere. This representative is found by

$$
v(x)=\lim _{r \rightarrow 0} f_{B(x, r)} u d y,
$$

which limit exists $p$-q.e. Moreover, $v$ is $p$-finely continuous $p$-q.e., that is, for $p$-q.e. $x \in \Omega$ there is a set $E$ such that $\left.v\right|_{\Omega \backslash E}$ is continuous at $x$ and $E$ is $p$-thin at $x$ (see [1, Thm. 6.4.5]). 
A very convenient characterization of $W_{0}^{1, p}(\Omega)$ functions is provided by the following:

2.1. The Havin-Bagby Theorem. Let $u \in W^{1, p}(\Omega)$. Then $u \in W_{0}^{1, p}(\Omega)$ if and only if there is a p-quasi continuous function $v$ on $\mathrm{R}^{n}$ such that $v=u$ a.e. on $\Omega$ and $v=0$-quasi everywhere on $\complement \Omega$.

If $p>n$, then $p$-quasi continuous functions are continuous whence it follows that for $p>n, u \in W_{0}^{1, p}(\Omega)$ if and only if there is a continuous function $v \in W^{1, p}\left(\mathrm{R}^{n}\right)$ such that $v=0$ on $\complement \Omega$ and $v=u$ a.e. in $\Omega$.

For these well known results we refer to the monographs [1], [2], [4], [13].

For technical reasons we shall use the relative $p$-capacity: for $E \subset \Omega$ the $p$ capacity of $E$ in $\Omega$ is the number

$$
\operatorname{cap}_{p}(E, \Omega)=\inf _{u} \int_{\Omega}|\nabla u|^{p} d x,
$$

where the infimum is taken over all $u \in W_{0}^{1, p}(\Omega)$ such that $u \geq 1$ on an open neighborhood of $E$.

The relative $p$-capacity admits the same null sets as the Sobolev $p$-capacity defined above. The relations to Hausdorff measures are well known: the Hausdorff dimension of a set of $p$-capacity zero does not exceed $n-p$, and if the $n$-p-dimensional Hausdorff measure of $E$ is finite, then $E$ is of $p$-capacity zero (see e.g. [1], [4], [2] etc.). We shall need the following density estimate (see [14] or [12]).

2.2. Lemma. Let $E \subset \mathrm{R}^{n}$. Suppose that $1 \leq p \leq n$ and $s>n-p$. Then

$$
\frac{\mathscr{H}_{\infty}^{s}(E \cap B(x, r))}{r^{s}} \leq c \frac{\operatorname{cap}_{p}(E \cap B(x, r), B(x, 2 r))}{r^{n-p}},
$$

where $c=c(n, p, s)>0$ and

$$
\frac{\operatorname{cap}_{p}(E \cap B(x, r), B(x, 2 r))}{r^{n-p}} \leq c \frac{\mathscr{H}_{\infty}^{n-p}(E \cap B(x, r))}{r^{n-p}},
$$

where $c=c(n, p)>0$.

Here $\mathscr{H}_{\infty}^{s}(E)$ is the $s$-Hausdorff content of the set $E$,

$$
\mathscr{H}_{\infty}^{s}(E)=\inf \left\{\sum_{i} r_{i}^{s}: E \subset \cup_{i} B\left(x_{i}, r_{i}\right)\right\} .
$$

We also employ a simple Hausdorff measure estimate that follows from a covering argument (see [2, p. 77]).

2.3. Lemma. Let $u \in L_{\mathrm{loc}}^{1}\left(\mathrm{R}^{n}\right)$ and $0 \leq s<n$. Then 


$$
\mathscr{H}^{s}\left(\left\{y \in \mathrm{R}^{n}: \limsup _{r \rightarrow 0} r^{-s} \int_{B(y, r)}|u| d x>0\right\}\right)=0 .
$$

Finally, we state a capacitary version of the Sobolev inequality, due to Maz'ya (an easy proof can be found in [5, 3.1], see also [13, 10.1.2] or [1, Cor. 8.2.7] and historical remarks there).

2.4. Lemma. Let $u$ be a p-quasicontinuous function in $W^{1, p}(B)$, where $B$ is a ball. There is a constant $c=c(n, p)>0$ such that

$$
\left(f_{B}|u|^{\kappa p} d x\right)^{1 / \kappa p} \leq c\left(\frac{1}{\operatorname{cap}_{p}(\{y \in B: u(y)=0\}, 2 B)} \int_{B}|\nabla u|^{p} d x\right)^{1 / p}
$$

where

$$
\kappa \leq\left\{\begin{array}{lll}
\frac{n}{n-p} & \text { if } & 1<p<n \\
2 & \text { if } \quad & p \geq n .
\end{array}\right.
$$

Of course, for $p \geq n \kappa$ could be any positive number in Lemma 2.4, but then the constant $c$ would depend on $\kappa$ too.

We close this section by proving that

$$
\Omega_{p}^{\text {dens }} \subset \Omega_{p} .
$$

Indeed, if $\overline{\operatorname{dens}}_{q}(\complement \Omega, x)>0$ for $q<p$, then $\overline{\operatorname{dens}}_{q}\left(\complement \Omega \backslash E_{p}, x\right)>0$ since $E_{p}$ is of $q$-capacity zero by the Kellogg property. Consequently, $\complement \Omega \backslash E_{p}$ is $q$-thick at $x$ whence it is $p$-thick. Thus we have

$$
e_{p}\left(\complement \Omega \backslash E_{p}\right) \cap \partial \Omega \subset\left\{x \in \partial \Omega: \overline{\operatorname{dens}}_{q}(\complement \Omega, x)=0\right\}
$$

and (2.5) follows.

\section{The first approach}

In this section we analyze the $p$-density condition $\Omega_{p}^{\text {dens }}$ and prove Theorem 1.2. We also present other results in this direction.

As discussed in the Introduction, the case where $p>n$ is settled so we are free to assume that $p \leq n$.

We first characterize $\Omega_{p}^{\text {dens }}$ in terms of Hausdorff measures. Employing the standard notation we write

$$
\theta_{\infty}^{* s}(E, x)=\limsup _{r \rightarrow 0} \frac{\mathscr{H}_{\infty}^{s}(E \cap B(x, r)}{r^{s}}
$$

for the upper $s$-density of the set $E$.

The following lemma makes it easy to check whether $\Omega$ is in $\Omega_{p}^{\text {dens }}$. 
3.1. Lemma. Let $1<p \leq n$. Then $\Omega \in \Omega_{p}^{\mathrm{dens}}$ if and only if for $p$-quasi every $x \in \partial \Omega$ there is $s>n-p$ with

$$
\theta_{\infty}^{* s}(\complement \Omega, x)>0 .
$$

Proof. This follows easily from Lemma 2.2. Indeed, Let $\Omega \in \Omega_{p}^{\text {dens }}$ and $x \in \partial \Omega$ such that $\overline{\operatorname{dens}}_{q}(\complement \Omega, x)>0$ for some $q<p$. Then by Lemma 2.2

$$
\theta_{\infty}^{* n-q}(\complement \Omega, x) \geq c \overline{\operatorname{dens}}_{q}(\complement \Omega, x)>0,
$$

and $s=n-q>n-p$.

On the other hand, if $s>n-p$ is such that $\theta_{\infty}^{* s}(\complement \Omega, x)>0$, choose $q$ with $n-s<q<p$ and obtain

$$
0<\theta_{\infty}^{* s}(\complement \Omega, x) \leq c \overline{\operatorname{dens}}_{q}(\complement \Omega, x),
$$

whence the lemma follows.

3.2. Remark. Belonging to $\Omega_{p}^{\text {dens }}$ is a rather mild condition. Indeed, for any domain $\Omega$ we have that the Hausdorff dimension of the set

$$
\Theta_{p}=\left\{x \in \partial \Omega: \theta_{\infty}^{* s}(\complement \Omega, x)=0 \text { for each } s>n-p\right\}
$$

does not exceed $n-p$ (see [3, 2.10.19]. The condition that $\Omega \in \Omega_{p}^{\text {dens }}$ would require that $\Theta_{p}$ is of $p$-capacity zero. Thus, if $\Omega \notin \Omega_{p}^{\mathrm{dens}}$, then $\Theta_{p}$ is of positive $p$-capacity and hence the Hausdorff dimension of $\Theta_{p}$ is exactly $n-p$ and $\mathscr{H}^{n-p}\left(\Theta_{p}\right)=\infty$.

Now we are ready to prove Theorem 1.2.

Proof of Theorem 1.2. Since $u \in W_{0}^{1, q}(\Omega)$ its zero extension is in $W^{1, q}\left(\mathrm{R}^{n}\right)$. In particular, $u$ (extended as zero) has distributional derivatives in $\mathrm{R}^{n}$ and therefore $u \in W^{1, p}\left(\mathrm{R}^{n}\right)$. Next we pick the $p$-quasi continuous representative of $u$. It is also $q$-quasi continuous. Using the Havin-Bagby theorem and the uniqueness of quasi continuous functions (i.e. two $q$-quasi continuous functions that agree a.e. on an open set agree in fact $q$-quasi everywhere) we infer that $u=0 q$-q.e. on $\complement \Omega$ for each $q<p$.

We claim that the estimate

$$
\liminf _{r \rightarrow 0} f_{B\left(x_{0}, r\right)}|u| d x \leq \limsup _{r \rightarrow 0} c\left(r^{p-n} \int_{B\left(x_{0}, r\right)}|\nabla u|^{p} d x\right)^{1 / p} .
$$

holds for $p$-q.e. $x_{0} \in \complement \Omega$ (here the constant $c$ may depend on the point $x_{0}$ ). Indeed, choose $x_{0}$ and $q<p$ such that $\overline{\operatorname{dens}}_{q}\left(\complement \Omega, x_{0}\right)>0$. Hence there are $\delta>0$ and a sequence of $r$ 's tending to zero so that

$$
r^{n-q} \leq \delta \operatorname{cap}_{q}\left(\complement \Omega \cap B\left(x_{0}, r\right), B\left(x_{0}, 2 r\right)\right) .
$$

Then we use the capacitary Sobolev inequality 2.4 to obtain 


$$
f_{B\left(x_{0}, r\right)}|u| d x \leq c\left(\frac{\int_{B\left(x_{0}, r\right)}|\nabla u|^{q} d x}{\operatorname{cap}_{q}\left(\left\{y \in B\left(x_{0}, r\right): u(y)=0\right\}, B\left(x_{0}, 2 r\right)\right)}\right)^{1 / q} .
$$

Since $u=0$ q-q.e. on $\complement \Omega$ we have

$$
\begin{aligned}
& \operatorname{cap}_{q}\left(\left\{y \in B\left(x_{0}, r\right): u(y)=0\right\}, B\left(x_{0}, 2 r\right)\right) \\
& \geq \operatorname{cap}_{q}\left(\complement \Omega \cap B\left(x_{0}, r\right), B\left(x_{0}, 2 r\right)\right) \geq \frac{r^{n-q}}{\delta}
\end{aligned}
$$

by (3.4). Consequently,

$$
f_{B\left(x_{0}, r\right)}|u| d x \leq c\left(r^{q-n} \int_{B\left(x_{0}, r\right)}|\nabla u|^{q} d x\right)^{1 / q} \leq c\left(r^{p-n} \int_{B\left(x_{0}, r\right)}|\nabla u|^{p} d x\right)^{1 / p}
$$

and (3.3) follows.

By Lemma 2.3 the right hand side of (3.3) is $0 \mathscr{H}^{n-p}$ a.e. and hence $p$-q.e. on $C \Omega$. Since $u$ is $p$-quasi continuous we thus obtain

$$
\left|u\left(x_{0}\right)\right|=\liminf _{r \rightarrow 0} f_{B\left(x_{0}, r\right)}|u| d x=0
$$

for $p$-q.e. $x_{0} \in \complement \Omega$. It follows that $u \in W_{0}^{1, p}(\Omega)$ by the Havin-Bagby theorem.

By repeating the above argument we obtain:

3.5. Corollary. Suppose that $1 \leq q<p$. If $\overline{\operatorname{dens}}_{q}(\partial \Omega, x)>0$ for p-quasi every $x \in \partial \Omega$, then

$$
W^{1, p}(\Omega) \cap W_{0}^{1, q}(\Omega)=W_{0}^{1, p}(\Omega) .
$$

The same holds if for $p$-q.e. $x \in \partial \Omega, \theta_{\infty}^{* s}(\complement \Omega, x)>0$ for some $s>n-q$.

REMARKS. i) If $\complement \Omega$ is uniformly $p$-fat, i.e. there is a constant $c$ such that

$$
\operatorname{cap}_{p}(\complement \Omega \cap B(x, r), B(x, 2 r)) \geq c r^{n-p}
$$

for all $r \leq r_{0}$ and $x \in \partial \Omega$, then by a theorem of Lewis [8] $\complement \Omega$ is uniformly $q$ fat for some $q<p$. Hence we have

If $\complement \Omega$ is uniformly $p$-fat, there is a number $q<p$ such that

$$
W^{1, p}(\Omega) \cap W_{0}^{1, q}(\Omega)=W_{0}^{1, p}(\Omega) .
$$

ii) It is easy to see that Lipschitz domains satisfy the condition of Corollary 3.5 for each $q \geq 1$. Furthermore this condition holds for each $q \geq 1$ if there is a corkscrew in $\complement \Omega$ at $p$-q.e. point on $\partial \Omega$ (i.e. for $p$-q.e. $x \in \partial \Omega$ there 
is a constant $c>0$ and a sequence $y_{j} \rightarrow x$ such that the balls $B\left(y_{j}, c\left|y_{j}-x\right|\right)$ do not touch $\Omega$ ). In both cases we conclude

$$
W^{1, p}(\Omega) \cap W_{0}^{1,1}(\Omega)=W_{0}^{1, p}(\Omega) .
$$

\section{The second approach}

In this section we prove the following theorem.

4.1. Theorem. Let $\Omega$ be a bounded open set in $\mathrm{R}^{n}$ and $1<q \leq p$. Then

$$
W^{1, p}(\Omega) \cap W_{0}^{1, q}(\Omega)=W_{0}^{1, p}(\Omega)
$$

if and only if $b_{q}(\complement \Omega)=\complement \Omega \backslash e_{q}(\complement \Omega)$ is $p$-thick p-q.e. on $\partial \Omega$.

Proof. Write $E=e_{q}(\complement \Omega) \cap \complement \Omega$ and observe that $E \subset \partial \Omega$. If the condition is not satisfied, then there is a compact set $K \subset \partial \Omega$ of positive $p$-capacity such that $\complement \Omega \backslash E$ is $p$-thin at each point of $K$. If $K \cap \overline{(\complement \Omega \backslash E)}=\emptyset$, then there is a ball $B$ such that $\bar{B} \cap \overline{(\complement \Omega \backslash E)}=\emptyset$, and $C_{p}(K \cap B)>0$. Any $w \in C_{0}^{\infty}(B)$ such that $w>0$ on a subset of $K \cap B$ with positive $p$-capacity satisfies $w \in W^{1, p}(\Omega) \cap W_{0}^{1, q}(\Omega)$, since $C_{q}(E)=0$ by the Kellogg property, but $w \notin W_{0}^{1, p}(\Omega)$, since $C_{p}(K)>0$ and $K \subset \partial \Omega$ Thus, we can assume that there is a compact $K$ as above, such that $K \subset \bar{\complement} \Omega \backslash E$. Invoking [1, Cor. 6.3.16] we find a ball $B$ and a bounded $p$-quasicontinuous function $v$ in $W^{1, p}\left(\mathrm{R}^{n}\right)$ such that

and

$$
\begin{gathered}
C_{p}(K \cap B)>0, \\
v=1 \text {-q.e. on } B \cap \complement \Omega \backslash E,
\end{gathered}
$$

$$
v<1 \text { on } K
$$

(the $(1, p)$-capacitary potential of $B \cap \complement \Omega \backslash E$ will do).

Next, let $w \in C_{0}^{\infty}(B)$ be such that $w>0$ on a subset of $K \cap B$ that has positive $p$-capacity. Then $w(1-v)=0 q$-q.e. on $\complement \Omega$ since $C_{q}(E)=0$ by the Kellogg property. Thus we infer from the Havin-Bagby theorem that

$$
w(1-v) \in W^{1, p}(\Omega) \cap W_{0}^{1, q}(\Omega),
$$

but

$$
w(1-v) \notin W_{0}^{1, p}(\Omega) .
$$

To prove the sufficiency let $u \in W^{1, p}(\Omega) \cap W_{0}^{1, q}(\Omega)$. As in the proof of Theorem 1.2 we see that $u \in W^{1, p}\left(\mathrm{R}^{n}\right)$ is $p$-quasicontinuous and $u=0 q$-q.e. 
on $\complement \Omega$. Moreover, $u$ is $p$-finely continuous at $p$-q.e. point in $\mathbf{R}^{n}$. Let $x_{0} \in \partial \Omega$ be such a point. If $x_{0} \notin E$, then the set

$$
\{y: u(y)=0\}
$$

is $q$-thick, whence $p$-thick, at $x_{0}$; thus $u\left(x_{0}\right)=0$ by $p$-fine continuity. It follows that $u=0 p$-q.e. on $\complement \Omega \backslash E$. Since by assumption, $\complement \Omega \backslash E$ is $p$-thick $p$ q.e. on $\partial \Omega$, the $p$-fine continuity yields $u=0 p$-q.e. also on $E$, whence $p$-q.e. on $\complement \Omega$. Thus $u \in W_{0}^{1, p}(\Omega)$ by the Havin-Bagby theorem.

Theorem 1.3 is proved in the same way. We leave the details to the reader.

The condition in Theorem 4.2 has many equivalent formulations; see [1, Theorems 11.4.1, 11.4.2]. We thus obtain the following corollary.

4.2. Corollary. Let $\Omega$ be an open bounded set in $\mathrm{R}^{n}$ and $1<q \leq p$. Then

$$
W^{1, p}(\Omega) \cap W_{0}^{1, q}(\Omega)=W_{0}^{1, q}(\Omega)
$$

if and only if one of the following conditions is satisfied:

(1) $C_{p}\left(G \cap b_{q}(\complement \Omega)\right)=C_{p}(G \cap \complement \Omega)$ for all open $G$;

(2) there is $\eta>0$ such that $C_{p}\left(G \cap b_{q}(\complement \Omega)\right) \geq \eta C_{p}(G \cap \complement \Omega)$ for all open $G$;

(3) $\liminf _{r \rightarrow 0} C_{p}\left(B(x, r) \cap b_{q}(\complement \Omega)\right) / C_{p}(B(x, r) \cap \complement \Omega)>0$ for $p$-q.e. $x \in \partial \Omega$;

(4) $e_{p}\left(b_{q}(\complement \Omega)\right)=e_{p}(\complement \Omega)$;

(5) $C_{p}\left(e_{p}\left(b_{q}(\mathrm{\complement} \Omega)\right) \backslash e_{p}(\mathrm{\complement} \Omega)\right)=0$.

Theorem 1.3 has a similar corollary, whose formulation we omit.

Observe that by the Kellogg property $\complement \Omega \backslash e_{q}(\complement \Omega)$ is always $p$-thick $p$-q.e. on $\partial \Omega \backslash e_{q}(\complement \Omega)$. Hence we obtain:

4.3. Corollary. Let $\Omega$ be a bounded open set in $\mathrm{R}^{n}$ and $1<q \leq p$. If $e_{q}(\complement \Omega) \cap \partial \Omega$ is of p-capacity zero, then

$$
W^{1, p}(\Omega) \cap W_{0}^{1, q}(\Omega)=W_{0}^{1, p}(\Omega) .
$$

The conclusion of Corollary 4.3 holds in particular when $\Omega$ is regular for the $q$-Laplacian Dirichlet problem since then $e_{q}(\complement \Omega) \cap \partial \Omega=\emptyset$ by [6].

\section{Stability of nonlinear eigenvalues}

In this section we apply Theorem 1.3 and discuss the stability of nonlinear eigenvalues. Our results complement those of Lindqvist [11]. We closely follow the terminology and notation of [11].

Let $\Omega$ be a bounded domain. The first eigenvalue $\lambda_{p}=\lambda_{p}(\Omega)$ of the $p-L a$ placian is the least real number $\lambda$ for which the equation

$$
\operatorname{div}\left(|\nabla u|^{p-2} \nabla u\right)+\lambda|u|^{p-2} u=0
$$


has a nontrivial solution $u$ in $W_{0}^{1, p}(\Omega)$, i.e. there is $u \in W_{0}^{1, p}(\Omega), u \neq 0$, such that

$$
\int_{\Omega}|\nabla u|^{p-2} \nabla u \cdot \nabla \varphi d x-\lambda \int_{\Omega}|u|^{p-2} u \varphi d x=0
$$

for all $\varphi \in C_{0}^{\infty}(\Omega)$; the nontrivial solution $u$ of (5.1) with $\lambda=\lambda_{p}$ is called the first eigenfunction of the $p$-Laplacian in $\Omega$. By approximation we may take any $\varphi \in W_{0}^{1, p}(\Omega)$ as a test function above. In particular the choice $\varphi=u$ yields

$$
\lambda_{p}=\frac{\int_{\Omega}|\nabla u|^{p} d x}{\int_{\Omega}|u|^{p} d x} .
$$

In fact, the first eigenvalue is the minimum of the Rayleigh quotient

$$
\lambda_{p}=\inf _{\substack{v \in W^{1, p}(\Omega) \\ v \neq 0}} \frac{\int_{\Omega}|\nabla v|^{p} d x}{\int_{\Omega}|v|^{p} d x} .
$$

This is seen as follows: using the weak compactness of $W_{0}^{1, p}(\Omega)$ and the Rellich-Kondrashov theorem we easily infer that there is a function $v \in W_{0}^{1, p}(\Omega),\|v\|_{p}=1$, that minimizes the Rayleigh quotient. Hence $v$ is a minimizer of the functional

$$
\int_{\Omega}|\nabla w|^{p} d x-\lambda \int_{\Omega}|w|^{p} d x
$$

whose Euler equation is (5.1) with $\lambda=\lambda_{p}$. Thus $v$ satisfies equation (5.1) and (5.2) holds.

Lindqvist proved in [10] that the first eigenvalue is simple, i.e. the ratio of any two (nontrivial) solutions of (5.1) with $\lambda=\lambda_{p}$ is constant. Furthermore, the first eigenfunction can be assumed to be positive, since $u$ can be replaced with $|u|$ in (5.2) and since eigenfunctions obey the minimum principle. This gives rise to the following normalization: we let $u_{p}$ stand for the first eigenfunction of the $p$-Laplacian in $\Omega$ such that $u_{p}>0$ and

$$
\int_{\Omega}\left|u_{p}\right|^{p} d x=1
$$

We shall consider the convergence of eigenvalues and eigenfunctions as $p$ varies. Let us recall the following [11, 3.4, 3.5]:

5.3. Lemma. For any domain the following limits exist and

$$
\lim _{q \rightarrow p-} \lambda_{q} \leq \lambda_{p}=\lim _{q \rightarrow p+} \lambda_{q} .
$$


In what follows we let

$$
\underline{\lambda}_{p}=\lim _{q \rightarrow p-} \lambda_{q}
$$

There is an example in [11] showing that it may happen that $\underline{\lambda}_{p}<\lambda_{p}$. We shall now analyze this phenomenon. We start by showing an easy trick to construct domains for which $\underline{\lambda}_{p}<\lambda_{p}$. First we prove an auxiliary result, well known for the ordinary Laplacian.

5.4. TheOrem. Let $D$ be a subdomain of $\Omega$. Then

$$
\lambda_{p}(D) \geq \lambda_{p}(\Omega) .
$$

Moreover, the equality holds if and only if $\Omega \backslash D$ is of p-capacity zero.

Proof. The monotonicity readily follows from the Rayleigh quotient (5.2). If $\Omega \backslash D$ is of $p$-capacity zero, then $W_{0}^{1, p}(\Omega)=W_{0}^{1, p}(D)$ by the Havin-Bagby theorem 2.1 and thus $\lambda_{p}(D)=\lambda_{p}(\Omega)$.

On the other hand, if $\lambda_{p}(D)=\lambda_{p}(\Omega)$ and if $u$ is the first normalized eigenfunction in $D$, then it follows from the Rayleigh quotient that $u$ is the first normalized eigenfunction also in $\Omega$. If $\Omega \backslash D$ is of positive $p$-capacity, then $u$ attains 0 at some point in $\Omega \backslash D$ by the Havin-Bagby theorem. This is absurd since the first eigenfunctions obey the minimum principle.

The following lemma can be used to create a lot of examples where $\underline{\lambda}_{p}<\lambda_{p}$.

5.5. Lemma. Suppose that that there is a domain $\tilde{\Omega} \supset \Omega$ such that $\tilde{\Omega} \backslash \Omega$ has positive p-capacity but zero q-capacity for each $q<p$. Then

$$
\lim _{q \rightarrow p-} \lambda_{q}(\Omega)<\lambda_{p}(\Omega) .
$$

Proof. By applying Theorem 5.4 twice we obtain

$$
\underline{\lambda}_{p}(\Omega)=\underline{\lambda}_{p}(\tilde{\Omega}) \leq \lambda_{p}(\tilde{\Omega})<\lambda_{p}(\Omega) .
$$

For instance, it follows from Lemma 5.5 that if $K$ is a Cantor set in any domain $\Omega$ such that $K$ has positive $p$-capacity and Hausdorff dimension $n-p$, then

$$
\underline{\lambda}_{p}(\Omega \backslash K)<\lambda_{p}(\Omega \backslash K) .
$$

The next result follows by mimicking the proof of $[11,3.12]$.

5.6. Proposition. Each sequence $q_{j} \leq p$ that converges to $p$ has a subsequence denoted again by $q_{j}$ with the property: there is a function 


$$
u \in W^{1, p}(\Omega) \cap \bigcap_{q<p} W_{0}^{1, q}(\Omega)
$$

such that $u_{q_{j}} \rightarrow u$ in $L^{p}(\Omega)$ and

$$
\lim _{j \rightarrow \infty} \int_{\Omega}\left|\nabla u_{q_{j}}-\nabla u\right|^{q_{j}} d x=0 .
$$

Moreover,

$$
\begin{array}{r}
\underline{\lambda}_{p}=\int_{\Omega}|\nabla u|^{p} d x, \quad \int_{\Omega}|u|^{p} d x=1, \\
\operatorname{div}\left(\left.\nabla u\right|^{p-2} \nabla u\right)+\underline{\lambda}_{p}|u|^{p-2} u=0,
\end{array}
$$

and

$$
\lambda_{p}=\inf _{\substack{v \in u+W_{0}^{1, p}(\Omega) \\ v \neq 0}} \frac{\int_{\Omega}|\nabla v|^{p} d x}{\int_{\Omega}|v|^{p} d x} .
$$

Putting this and $[11,3.6]$ together with the uniqueness of the first eigenfunction we immediately obtain:

5.7. Corollary. Suppose that

$$
W_{0}^{1, p}(\Omega)=W^{1, p}(\Omega) \cap \bigcap_{q<p} W_{0}^{1, q}(\Omega) .
$$

Then

$$
\lambda_{p}=\lim _{q \rightarrow p} \lambda_{q}
$$

and

$$
\lim _{q \rightarrow p} \int_{\Omega}\left|\nabla u_{q}-\nabla u_{p}\right|^{\tilde{q}} d x=0
$$

where $\tilde{q}=\min (q, p)$.

Hence Theorem 1.3 yields

5.8. Corollary. If $\Omega \in \Omega_{p}$, then

$$
\lambda_{p}=\lim _{q \rightarrow p} \lambda_{q} .
$$

In particular, if $p>n$, then 


$$
\lambda_{p}=\lim _{q \rightarrow p} \lambda_{q}
$$

is true for all bounded domains.

It remains in doubt whether $\Omega \in \Omega_{p}$ is a necessary condition to have $\lambda_{p}=\underline{\lambda}_{p}$.

We finish this paper by giving a uniform convergence result that is a straightforward consequence of Corollary 5.7, Theorem 1.2 and $[11,6.3]$.

5.9. THEOREM. If $\Omega \in \Omega_{p}$ and $q_{j}$ is any sequence converging to $p$, then $u_{q_{j}} \rightarrow u_{p}$ and $\nabla u_{q_{j}} \rightarrow \nabla u_{p}$ locally uniformly in $\Omega$.

Proof. By $[11,6.3]$ there is a subsequence of $q_{j}$ such that $u_{q_{j_{i}}} \rightarrow u$ and $\nabla u_{q_{j_{i}}} \rightarrow \nabla u$ locally uniformly in $\Omega$. By 1.2 and 5.7 the limit function is necessarily $u_{p}$. Hence, the limit is independent from the choice of the subsequence and the theorem follows.

\section{REFERENCES}

1. D. R. Adams and L. I. Hedberg, Function Spaces and Potential Theory, Springer, 1996.

2. L. C. Evans and R. F.Gariepy, Measure theory and fine properties of functions, CRC Press, 1992.

3. H. Federer, Geometric Measure Theory, Springer, 1969.

4. J. Heinonen, T. Kilpeläinen and O. Martio, Nonlinear Potential Theory of Degenerate Elliptic Equations, Oxford University Press, Oxford, 1993.

5. T. Kilpeläinen and P. Koskela, Global integrability of the gradients of solutions to partial differential equations, Nonlinear Analysis, TMA 23 (1994), 899-909.

6. T. Kilpeläinen, and J. Maly', The Wiener test and potential estimates for quasilinear elliptic equations, Acta Math. 172 (1994), 137-161.

7. J. Kinnunen and O. Martio, Hardy's inequalities for Sobolev functions, Math. Res. Lett. 4 (1997), 489-500.

8. J. L. Lewis, Uniformly fat sets, Trans. Amer. Math. Soc 308 (1988), 177-196.

9. G. Li and O. Martio, Stability of solutions of varying degenerate elliptic equations, Indiana Univ. Math. J. 47 (1998), 873-891.

10. P. Lindqvist, On the equation $\operatorname{div}\left(|\nabla u|^{p-2} \nabla u\right)+\lambda|u|^{p-2} u=0$, Proc. Amer. Soc. 109 (1990), 157-164; Addendum, Ibid. 116 (1992), 583-584.

11. P. Lindqvist, On non-linear Rayleigh quotients, Potential Analysis 2 (1993), 199-218

12. O. Martio, Capacity and measure densities, Ann. Acad. Sci. Fenn. Ser. A I. Math. 4 (1978/ 1979), 109-118.

13. V. G. Maz’ja, Sobolev Spaces, Springer-Verlag, 1985.

14. Yu. G. Reshetnyak, The concept of capacity in the theory of functions with generalized derivatives, Sib. Mat. Zh. 10 (1969), 1109-1138, (Russian). English transl. in Siberian Math. J. 10 (1969), 818-842.

DEPARTMENT OF MATHEMATICS

LINKÖPING UNIVERSITY

SE-58183 LINKÖPING

SWEDEN

e-mail: lahed@mai.liu.se
DEPARTMENT OF MATHEMATICS UNIVERSITY OF JYVÄSKYLÄ

P.O. BOX 35

FIN-40351 JYVÄSKYLÄ, FINLAND

e-mail: terok@math.jyu.fi 